\title{
APPLICABILITY OF THE OODA LOOP THEORY FOR ANALYSIS OF HUMAN ERROR PROCESSES IN THE MARITIME SAFETY
}

\author{
Boyan Mednikarov, Siyana Lutzkanova \\ Nikola Vaptsarov Naval Academy (Bulgaria)
}

\begin{abstract}
The publication evaluates the applicability of John Boyd's OODA Loop (observe-orient-decide-act) theory for the analysis of human error in the maritime industry. The predominant share of human error among the range of maritime safety issues is revealed. The interdependence between the different phases of the OODA Loop to achieve the desired state of maritime safety is studied, namely the correct actions in each of the implemented cycles. So, the paramount importance of situational awareness as a prerequisite for safety is proven. The mechanisms for manifestation of other factors endangering maritime safety are also analyzed, such as: lack of competence, fatigue, stress, etc. In conclusion, the role of the time factor in the realization of the OODA Loop is assessed and in this context, approaches are offered to increase the possibility for realization of a quality loop.
\end{abstract}

Keywords: OODA Loop; human error; leadership training; maritime safety

\section{Introduction}

It is estimated that between $74 \%$ and $95 \%$ of marine accidents can be associated with human error. Human error may occur in different situations and is subject to broad and deep interdisciplinary research. The human factor is a key element in the high risk working environments where knowledge, experience, fast decision making abilities, leadership skills and even physical resistance can affect the outcome of incidents at sea related to security and safety issues.

Most of the statistics and published studies have concluded that maritime accidents are caused mainly by human errors. Incidents caused by maritime security issues (deliberate human interference to cause extensive damage to human life, material losses or ecological catastrophe) should be analyzed in the context of the acting regulations and procedures for planning, organizing and maintaining shipping and port security in the current dynamic and unpredictable maritime security environment on global, regional and national levels due to the different security factors that could occur. So, the common approach involving the role of the human factor in maritime security issues are the scenario-based theories 
where different kind of maritime operations (interdiction operations, maritime security operations, etc.) aiming at taking protective measures against threats to merchant ships and port facilities. Another implemented approach deals with the risk management involving threat mitigation, security equipment, coordination and interaction, planning and contingency planning.

This paper focuses primarily on maritime safety issues and the processes of human error in the context of identifying the most common causes of marine accidents due to human failure and the applicability of one of the broad implemented explanation theories for mitigation of that risky human behavior that stems from the military science - the OODA Loop of Colonel John Boyd.

\section{Causes for marine incidents}

In many cases, the crew is the target of fault even before the investigations are concluded. Human factors occur in many cases but the master and the crew are not always the main cause for negative outcome of an accident. Recent studies go beyond this point and examine whether errors are made by members of the crews of the damaged ships or by other involved parties like pilots, shore and port personnel, company staff, etc. The adequate classification of human error provides additional information on the integration between bridge team and other assisting staff. Another point is the environment in which the ship is sailing, stated that most of the accidents occur in narrow waterways due to limited maneuverability or in intensive maritime traffic that requires more challenging navigation skills from pilot and crew (Sánchez-Beaskoetxea et al. 2021).

According to J. Sánchez-Beaskoetxea et al. based on extensive research obtained from the analysis of 225 accident reports, involving 264 ships, submitted to the National Transportation Safety Board (NTSB) from 1975 to 2017 , the percentage of human error caused by the crew is $45.83 \%$ of all accidents analyzed, including the cases where more than one ship is involved in the incident. Therefore, human error in ship accidents is not always a crew error. Interesting conclusions are drawn when the type of ship is considered. Human error in cargo/passenger ships is around $82 \%$, but crew error is only $43 \%$. Pilot error is responsible for $43 \%$ of total cases with other people's error in this type of vessel (Sánchez-Beaskoetxea et al. 2021, 7).

Navigation is not the exclusive cause of marine accidents. Another influential factor in the joint error of pilot and crew. Disintegration in the bridge team due to ineffective bridge resources management is pointed out (Park et al., 2019). Pilot errors are associated mainly with poor communication that requires additional measures rather than only exchange of information between both for a safe transit.

Regarding the types of errors, the study points to navigation as the primary cause. However, navigation errors are caused mainly by wrong judgements and 
overconfidence. Latest accidents show over-reliance on electronic devices and the electronically provided data. Training for officers on watch to try to reduce overconfidence is necessary. Other errors, ordered by frequency, are inadequate planning, poor communication, physical problems and distractions (SánchezBeaskoetxea et al. 2021, 7-8).

So, complementing the well-known "indicators" for error chain development from the main question is whether and to which extent the main assumptions of the OODA loop could be applied to break the error chain connected to the majority of marine accidents based on disrupted interaction with the environment.

\section{Why use the OODA Loop in the maritime environment}

The Boyd concept hypothesize that all intelligent organisms and organizations undergo a continuous cycle of interaction exactly with their environment consisting of four interrelated processes.

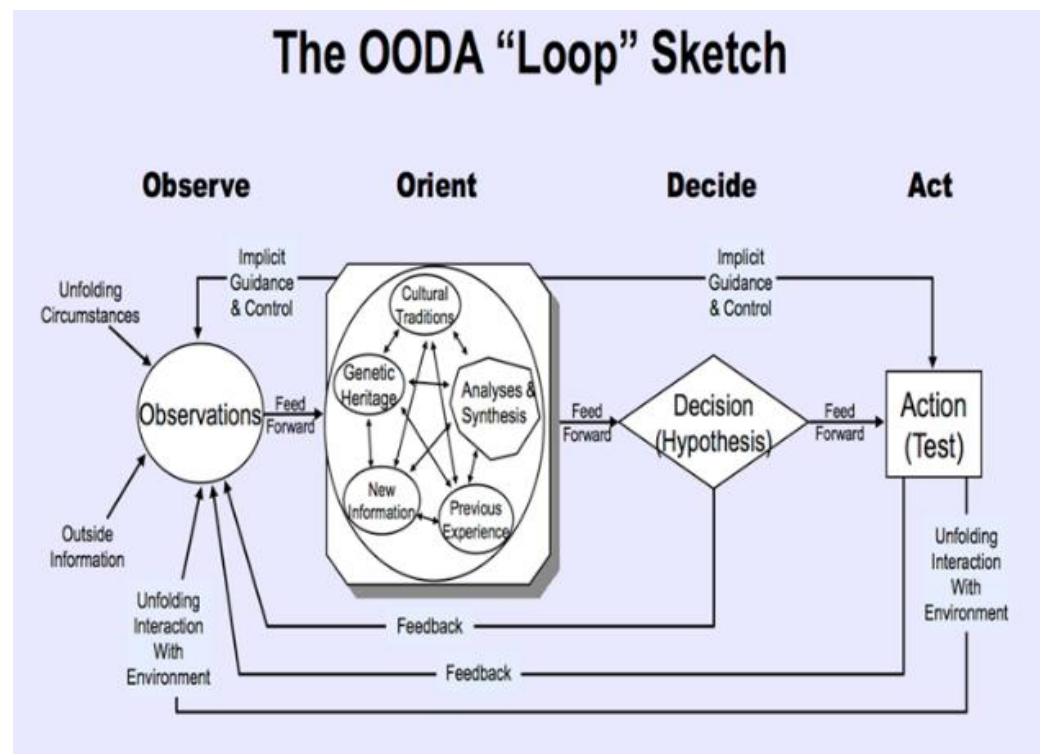

Figure 1. The OODA "Loop" Sketch

The OODA Loop was Boyd's attempt to explain how we create mental patterns or "concepts of meaning" in order to be able to comprehend and cope with our environment. Boyd described this approach as an attempt "to sketch out how we destroy and create these patterns to permit us to both shape and be shaped by a changing environment". In this sense, "we cannot avoid this kind of activity if we intend to survive on our own terms" (Boyd 1996). 


\begin{tabular}{|l|l|l|l|l|}
\hline $\begin{array}{l}\text { OODA } \\
\text { Loop }\end{array}$ & Observation & Orientation & Decision & Action \\
\hline $\begin{array}{l}\text { Situational } \\
\text { Awareness }\end{array}$ & Perception & $\begin{array}{l}\text { Comprehension/Pro- } \\
\text { jection }\end{array}$ & Decision & Action \\
\hline $\begin{array}{l}\text { Risk Ma- } \\
\text { nagement } \\
\text { Cycle }\end{array}$ & Monitor & Identification & Analysis & $\begin{array}{l}\text { Respon- } \\
\text { se }\end{array}$ \\
\hline $\begin{array}{l}\text { Shewhart } \\
\text { Cycle }\end{array}$ & Planning & Action & $\begin{array}{l}\text { Chec- } \\
\text { king }\end{array}$ & Action \\
\hline $\begin{array}{l}\text { Six Sigma } \\
\text { DMAIC } \\
\text { Cycle }\end{array}$ & $\begin{array}{l}\text { Define/Mea- } \\
\text { sure }\end{array}$ & Analyze & Improve & Control \\
\hline
\end{tabular}

Figure 2. OODA Loop and other Cycles

A key difference between the OODA Loop and other cycles lies in the numerous continuous feedback and feed forward connections that are identified within the full Boyd diagram (Gray et al. 2015). The OODA Loop suits and complements the situational awareness concept (SAW). This is a cyclic process that gives the background of situational awareness concept to be related to decision making (Niklasson et al. 2008; Endsley 2008). Situational awareness is the result from situation analysis (SA). Situation analysis is a process; situational awareness (the state of mind) is the result of this process.

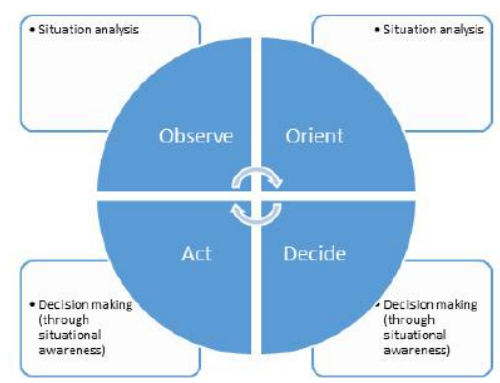

Figure 3. The OODA Loop in relation to SA and SAW

Vital for situation analysis is the pick-up of only the relevant information related to the situation. When the decision maker has become aware of the current situation and its future implications, the actual decision, respectively action, might be correctly taken. Thus, the situation analysis covers the Observe and Orient steps, resulting in decision-making process and action (situational awareness).

The availability of huge amounts of information does not mean that we can incorporate it or capitalize on it in a decision making process. Proper tools and automatic assistance will often be necessary, especially when under time pressure. 
(Niklasson et al. 2008, 3) For certain tasks, automated decision support either supports human decision making or fully substitutes it. For complex tasks and environments in the maritime, such fully automated situation analysis is not proper due to security and safety reasons but integration between machine and human analysis is feasible.

As far as the OODA Loop concept describes decision making as a mental issue (even on abstract level), it considers the complexity, the inter-connections and relationships between the described four steps similar to the competing priorities that exist between shipping company owners, maritime network administrators, and external partners or service providers. Activities that happen and act in such complex and dynamic environment must prioritize their efforts. OODA Loop allows the subjects of the maritime industry to focus on those steps necessary to improve abilities to shape and adapt to different circumstances.

By "orientation", we consider "analysis of information" and the creation of an idea of what is happening around us, what is our perspective on seeing things. Here, under "perspective", we are not considering directly something that is happening in the future, but how we see things. When we talk about "orientation", we can have complete reliable information and then we will say - something is happening, we see clearly what is happening, but very often we can have some problems with orientation, problems with the information that we get and then we can make only hypotheses.

From this point of view, when we make decisions, if the information is clear - the decision is clear as well. But, if we have hypotheses, then we should also make hypotheses from the point of view of decisions, if it is under option 1 - such a decision; if it is under option 2 - another decision, etc. Once we make decisions, the action follows. This is Boyd's assumption - that all intelligent beings, but also organizations, are constantly realizing this cycle of interaction with their environment. Again, here is the idea that systems do not exist in a vacuum. They are always in the middle of a bigger environment.

The "observation" process is the collection of data through our senses. The process of "orientation" or "assessment" is an analysis of these data, depending on their completeness and forming an idea of what is happening in the situation. The solution is to determine the course of action based on the situation. The action is the physical realization of our decision.

To get safely from one point to another, in the "observation" phase, we receive the information with our senses. In the "orientation" phase, we analyze this information. We see what is most important, we focus on that and we analyze, interpret this information to get an idea of the situation - our understanding of the situation. In the next "decision" phase - based on our orientation, based on our perception of the situation, we make our decision and in the "action" phase - we act. Speaking of action, we should consider what are the possibilities for action. There is a glass in front of us and we move it to the next table, i.e. we performed a 
physical manipulation. This is an action - there is a glass and we stand with folded arms, waiting for our assistant, who helps us in our work, to pick it up. So, waiting is an action. Since we apply this concept in the maritime industry, we are talking about chain of command. Very often, a senior officer's actions are not specific physical manipulations, but they are related to performing someone else physical actions, e.g. giving orders and other people will perform specific physical actions following the given instructions.

In fact, through the process of "observation" we begin to understand what is happening around us. Through the process of "orientation" we define the situation - what happens. Through the process of "decision" we create the model, the design of what we have to do and through the process of "action" we de facto perform our specific actions. We implement our solution.

The OODA process is a cycle. The OODA Loop also controls the ship. The watch officer observes what is happening around him, he evaluates the situations that arise. Based on this assessment, he must decide whether to change the course, the speed of the ship, whether to communicate with the other ship, and then action must follow. In fact, what happens in terms of this theory when a situation has changed. The result is a second OODA cycle. We decide that the situation is no longer safe, we assess the situation anew and decide again. Each new cycle corrects the previous one, and the correction can be in such a direction: the first cycle was wrong, but the second is true; the first was wrong and the second was wrong; the first cycle is true and the second is true and the first cycle was true and the second was false, it means all combinations are possible. From this point of view, it will be feasible to guarantee the quality of each OODA Cycle. How to make sure that we always manage to perform a normal cycle? A normal OODA cycle is that one which ends with the right actions.

Boyd's theory, from the point of view of organizations, says that large organizations, corporations, governments, the Armed Forces, companies - they have systems or hierarchies of OODA Loops as well. They are implemented at different levels. From the executive director of the shipping company, the flotilla director, to the captain and watch officer onboard. OODA Loops run at different levels, at the same time in parallel. And, if there is good communication within the company, the effects of the OODA Loops reach everyone in the organization.

\section{Applicability of OODA Loop in breaking "the chain of errors". Safety in the context of OODA cycles}

Safety in terms of this theory means that every cycle should end with the right action. A mistake, an error happens when the cycle ends with a wrong action. This includes the lack of proper action as well, e.g. whether we waited, or we should not have waited - it is perceived as a wrong action. Of course, giving the wrong order/ instruction would be the wrong action too. 
A logical connection seems important in this context: Can we have the right action if our decision was wrong? The probability of having the right action following wrong decision is very low. This can only happen if, while performing the action, some dramatic changes occur in the situation. This is already changing the context - suddenly the wrong decision begins to turn out to be right followed by the right actions. Assuming that this is possible, but with a very low probability can we have the right decision if our assessment, if our orientation is wrong? The probability is low, even lower than the previous one. The question is - not whether we have information or not, its evaluation depends on how we understand and interpret it. We may have a lot of information, but we are not able to evaluate it because of a lack of knowledge. Existence and sharing of the information alone means nothing without the proper knowledge to understand and interpret it. Obviously, we need the right assessment, followed by the right decisions and the right actions. Can we have a correct assessment if we receive wrong information? If we see the situation around us wrong? If we have wrong information, we cannot imagine the specific situation and find a solution to the specific problem.

If we are looking for the probability of how to go from the wrong observation to the right decision, this probability will be a product of the previous three probabilities described above. However, in all three cases the probability is low, even lower, and eventually possible only if intuition is present. In fact, from the point of view of safety, if we interpret it through the theory of the OODA loop, it turns out that the most important thing is to see the picture correctly, to see correctly what is happening around us. This is added to all those causes of error - stress, lack of knowledge, alcohol, fatigue, etc.

The largest share of human errors in marine incidents is given to the situational orientation, and the second to situational assessment. In fact, these are the first two phases of the OODA cycle. That seems to be a key point to safety problems.

Humans are not able to see the surrounding world 100\% objectively. The human senses - eyes, taste, smell, hearing and touch - do receive only some information from the world. This information reaches our consciousness and our brain. What will our assessment depend on? It will depend on our worldview, our knowledge, our experiences, our life, etc. This is definitely not objective, it is subjective and there is no way for each person to have the same understanding. Different experiences constantly shape and mould our worldview. It is constantly changing as well, the more we know the more we can do. The differences and deviations in professional, highly specialized groups will be small, but there will still be some deviations!

The captain is not in the engine room, the captain is on the bridge when a fire occurs. How does the captain see the fire? How does he get the information about the fire? Through reports. The one who reports could distort the information, not on purpose and not deliberately. If the one who reports passes wrong information, then the master is given wrong information, the observation is wrong and the assessment 
is wrong. Decisions and actions seem wrong as well. Attention should be paid to situational orientation and situational assessment as factors to deal with the surrounding world without error. Here, factors like fatigue, lack of sleep, alcohol, etc., do limit and damage every phase of the OODA cycle resulting in error.

In maritime safety, the role of stress, especially the so-called "bad stress" is particularly important. We generally associate distress with the feeling of losing control of the situation, with the fear of what is happening. In fact, this is equivalent to the fact that an irritant appears and we have to share all our mental resources to evaluate, analyze what is happening in the situation. Loss, fears, shocks block our abilities to control the situation. This is associated with the well-known Maslow Pyramid. Abraham Maslow, in the 60s of the twentieth century, created his theory of Meta motivations. He graded human needs on five levels: the most important human needs are at the base of the pyramid - these are the physiological needs, the second level is the need for security and safety, the third - belonging, love, etc. The fifth is self-realization. The principle of Maslow's pyramid is also that if we have a deficit at a lower level, at a more primary, basic level - this automatically leads to a deficit at the next levels. By physiological needs we mean in the first place - to breathe, to eat, to drink water, to sleep, to be in temperature comfort, etc. When we talk about bad stress, it's essentially Maslow's second-level deficit. We have a problem because we have lost control of the situation, we feel insecure and restless. This is bad stress, including different kind of deprivations that may improve the negative stress feeling.

To conclude, maritime accidents are very rarely the result of a single event. They tend to arise from a series of different reasons. Control of the situation, i.e. knowing what is going on around the ship, will help the watch officer to determine that the chain of errors is evolving, requires increased attention and adequate action based on accurate information to break this chain. Some signs in the activities of the bridge team show that the chain of errors is evolving. This does not mean that the accident almost happened, but the transition is not going according to plan, the ship is involved in unnecessary risk, so action must be taken to break the chain of errors. For example, distraction can occur from overwork, stress or fatigue, emergency conditions or inattention to detail, from surprises that do not endanger the ship, such as calling a UVV radio station that takes the person's entire attention. Another example is when independent warnings about the location of the ship from GPS and radar give different "points", obviously some of them are wrong. A less conscientious watch officer will simply notice this fact, while another will try to determine why there was a difference. There may be two or more personal opinions about the decision-making of several crew members. A demoralized team or even a member of the team will not be able to perform their duties in accordance with high safety standards. Morale depends on many reasons, but the coordinated and effective work of the team will improve if each member of the team clearly presents 
his role in it and sees the result of his work, noticing on time the shortcomings in their work and the ability to correct mistakes in advance before the moment when the situation might become critical (Yanchuk 2003).

OODA Loop includes the context of the time factor. The crew and the ship's traffic control system operate at different time limits. Decision time varies from fractions of a second in emergencies, when the task can be performed only through active self-regulation and maximum use of special and professional potential, up to many minutes. According to research conducted by psychologists on different types of ships, the complexity of the task at a certain level does not create any difficulties for the bridge crew. According to situational requirements, it adapts to the new organization of the surveillance service, and the captain easily controls the rhythm of his own actions and the actions of his subordinates.

When the time limits the ability to choose options, the master rearranges the tactics of his work with the bridge team, mobilizing operational thinking, raising the threshold of sensitivity and selectivity to the signals corresponding to the task. Such mobilization of activities happens involuntarily, without the active will of the captain. This situation requires much more energy and information support. However, his activities are already classified as mentally stressful.

In situations at night, in unfavorable climatic and meteorological conditions, in the event of complex navigational situations, the decision-making period reaches a level where forced self-regulation does not give the desired effect, and quality indicators actually begin to decline. In order to maintain them at the required level, the captain, as the team leader, forces active voluntary regulation, which makes his work and the team's even more stressful. In situations of congestion, canals, port visits, the solution of the navigation task should be done not on standard characters, but with the number of variables that correspond to the actual number of ships, coastal means, YPG and navigation signals. The captain and crew then begin to manage the system selectively, focusing mainly on achieving the most important results and avoiding side effects. To conclude, time factor strengthens and challenges the proper passing through the OODA cycle.

\section{Conclusion}

The recently identified most common marine incident' causes that are connected with disrupted feedback to and from the environment make the OODA Loop an effective model for understanding complex challenges in the maritime environment. This includes specific abilities in knowing what is going on around the ship by recognizing that an error chain is developing and taking adequate actions on time to break it; the right perception of factors and conditions affecting the operation of the vessel (physical conditions, traffic situation, communication); and continuous monitoring of the environment to enable the recognition of danger situation. Important issues are stress and distress management techniques, proper 
coordination and communication approaches in the crew that minimize the negative effect of various personal or professional differences.

\title{
REFERENCES
}

Sánchez-Beaskoetxea, J., Basterretxea-Iribar, I., Sotés, I., Maruri Machado, M., 2021. Human error in marine accidents: is the crew normally to blame? Maritime Transport Research Journal 2. Available from: https:// www.sciencedirect.com/science/article/pii/S2666822X21000083?via\% 3Dihub

Park, Y.A., Yip, T.L., Park, H.G., 2019. An analysis of pilotage marine accidents in Korea. Asian J. Shipping Logist. 35(1), 49-54.

Boyd, J.R., 1996. The Essence of Winning and Losing. Unpublished lecture notes.

Gray, D., et al., 2015. Improving Federal Cybersecurity Governance Through Data-Driven Decision Making and Execution, 6-7. Available from: https://www.researchgate.net/publication/281843717 Improving_Federal_Cybersecurity_Governance_Through_Data-Driven_Decision_Making_and_Execution.

Niklasson, L., et al., 2008. Extending the scope of situation analysis. Available from: https://www.researchgate.net/publication/4370007_Extending the scope_of situation_analysis

Endsley, MR., 2008. Design and evaluation for situation awareness enhancement. In: Proceedings of the Human Factors Society 32nd Annual Meeting, 97-101. Santa Monica, CA: Human Factors Society

Yanchuk, V.A., 2003. Social Psychology. Moscow: APO.

\author{
Boyan Mednikarov \\ https://orcid.org/0000-0003-4247-897X \\ Nikola Vaptsarov Naval Academy \\ Varna, Bulgaria \\ E-mail: b.mednikarov@naval-acad.bg \\ $\triangle$ Siyana Lutzkanova \\ https:// orcid.org/0000-0003-0530-7397 \\ Nikola Vaptsarov Naval Academy \\ Varna, Bulgaria \\ E-mail: s.lutzkanova@nvna.eu
}

